scanning must be assessed, and we are committed to ongoing analysis and re-evaluation.

\author{
P.A. de Jong*,\#, M.H. Lequin ${ }^{\top}$, J.R. Mayo ${ }^{+}$, P.D. Paré\#, \\ H.A.W.M. Tiddens* \\ Depts of *Paediatric Pulmonology, and "Paediatric Radiology, \\ Erasmus MC-Sophia, Rotterdam, The Netherlands. ${ }^{\text {UBC }}$ \\ James Hogg iCAPTURE Centre for Cardiavascular and \\ Pulmonary Research, St Paul's Hospital, and ${ }^{+}$Dept of \\ Radiology, Vancouver Hospital and Health Science Center, \\ Vancouver, BC, Canada.
}

\section{References}

1. de Jong PA, Nakano Y, Lequin MH, et al. Progressive damage on high-resolution computed tomography despite stable lung function in cystic fibrosis. Eur Respir J 2004; 23 : 93-97.

2. European Council Directive 97/43/Euratom and repealing Directive 84/466/Euratom. The Medical Exposures Directive. europa.eu.int/comm/energy/nuclear/radioprotection/ doc/legislation/9743_en.pdf.

\title{
Variable phenotype associated with SP-C gene mutations: fatal case with the I73T mutation
}

\section{To the Editors:}

We read with great interest the report by BRASCH et al. [1] on a case of interstitial lung disease (ILD) due to a de novo heterozygous missense mutation in the surfactant protein-C gene (SFTPC), which resulted in the substitution of threonine for isoleucine in codon 73 of the propeptide of the surfactant protein (SP)-C proprotein (I73T). Histological findings were consistent with nonspecific interstitial pneumonia (NSIP) and pulmonary alveolar proteinosis (PAP) features. Therapy included whole-lung lavages and anti-inflammatory drugs, and the child is still alive.

The natural history of lung disease associated with SP-C mutations is poorly characterised, with unpredictable shortand long-term outcomes. In order to emphasise the phenotypic variability of SFTPC mutations, we now report a child with fatal lung disease due to the SP-C I73T mutation. The child was a full-term male, born to unrelated parents from an uneventful twin dizygotic pregnancy. At 3 months, he developed episodes of asthmatic bronchitis. At 9 months, he was admitted to the hospital because of progressive failure to thrive and moderate-to-severe delay in neuromotor development that had started at 5 months. Dyspnoea and tachypnoea were observed, and arterial oxygen tension and arterial carbon dioxide tension in room air were $39 \mathrm{mmHg}$ and $37 \mathrm{mmHg}$, respectively, and $\mathrm{O}_{2}$ supplementation was started. Chest imaging findings, including conventional radiograph plus high-resolution computed tomography, were consistent with diffuse ILD. Cystic fibrosis, respiratory infectious diseases, lysinuric protein intolerance with respiratory failure due to PAP, gastro-oesophageal reflux disease, and defects in the immune system were excluded [2-4]. A thoracoscopic lung biopsy was performed when the child was 11 months old. Histology showed widened alveoli with thickened alveolar septa infiltrated mainly by fibroblasts and lymphocytes, hyperplasia of type-II pneumocytes, and massive intraalveolar accumulation of finely granular periodic acidSchiff-positive material and several cholesterol crystals, consistent with NSIP and PAP features.

An SP abnormality was suspected. Hereditary SP-B deficiency was unlikely, given the usually rapidly fatal nature of that disorder, and, moreover, immunohistochemical staining for SP-B was detected. Genetic analysis revealed a heterozygous mutation ( $\mathrm{T}$ to $\mathrm{C}$ transition in position 1286), resulting in a substitution of threonine for isoleucine (I73T) in the SFTPC. Neither parent carried this mutation, consistent with a de novo mutation resulting in sporadic disease. The mutation in the presence of ILD and the absence of the mutation and disease in the parents further strengthened the association between the genetic abnormality and the disease.

During the following 4 months, the infant often needed antibiotics because of recurrent respiratory infections, in addition to a slowly progressive need for $\mathrm{O}_{2}$ supplementation. Oral steroid treatment was started, along with evaluation for lung transplantation. Unfortunately, 3 months later, he developed acute respiratory failure associated with a possible bacterial respiratory infection, which required admission to the Paediatric Intensive Care Unit (Cardarelli Hospital, Naples, Italy). Despite mechanical ventilation, his condition progressively worsened, and the child died 8 months postbiopsy. The parents did not authorise an autopsy.

In the last few years, several mutations for the SFTPC have been identified [1, 5-10]. Recently, HAMvAs et al. [10] described an infant with a progressively severe ILD who successfully underwent lung transplantation. In this case, a spontaneous heterozygous nine base-pair deletion was identified in the exon 3 of the SFTPC, which is in the same general location in which BRASCH et al. [1] and our group demonstrated the g.1286T $>C$ transition.

Information describing the clinical expression of SFTPC mutations is accumulating, and it is clear that such mutations can cause ILD in children and adults. The histological expression of mutations associated with SFTPC is pleiotropic, and ranges from chronic pneumonitis of infancy and nonspecific ILD in children to usual or desquamative interstitial pneumonia and idiopathic pulmonary fibrosis in adults [11]. Moreover, age at presentation is quite variable, with some children developing symptoms in early infancy, and others not until adulthood, and some remaining symptom free. As the same specific mutations can be associated with variable phenotypic expression, modifier genes or interactions between genetic background and environmental factors are likely to be important in modifying the course of the disease $[8,9]$.

Further specific information about clinical manifestations, histological appearance and genetic basis related to surfactant protein-C abnormalities are needed in order to provide an explanation for familial and sporadic cases of interstitial lung disease that may remain otherwise undiagnosed. Since the clinical course may be characterised by periods of substantial improvement, followed by others with more severe activity triggered by infections eventually leading to fatal respiratory failure, a better understanding of what modifies or exacerbates the course of this disease might provide some valuable clues on how to better treat it. Finally, given the variability of phenotypic expression of the lung disease related to surfactant 
protein- $\mathrm{C}$ gene mutations, neonatologists and paediatricians should consider a surfactant protein- $\mathrm{C}$ abnormality as a possible diagnosis in a young infant with interstitial lung disease or an older patient with difficult-to-interpret lung histology.

S. Percopo*, H.S. Cameron ${ }^{\#}$, L.M. Nogee ${ }^{\#}$, G. Pettinato", S. Montella*, F. Santamaria*

Depts of *Paediatrics and 'Biomorphological and Functional Sciences, Federico II University, Naples, Italy. "Dept of Paediatrics, Johns Hopkins University School of Medicine, Baltimore, MD, USA.

\section{References}

1. Brasch F, Griese M, Tredano MZ, et al. Interstitial lung disease in a baby with a de novo mutation in the SFTPC gene. Eur Respir J 2004; 24: 30-39.

2. Santamaria F, Brancaccio G, Parenti G, et al. Recurrent fatal pulmonary alveolar proteinosis following heart-lung transplantation in a child with lysinuric protein intolerance. J Pediatr 2004; 145: 268-272.

3. Langston C, Fan LL. Diffuse interstitial lung disease in infants. Pediatr Pulmonol 2001; S23: 74-76.
4. Trapnell BC, Whitsett JA, Nakata K. Pulmonary alveolar proteinosis. $N$ Engl J Med 2003; 349: 2527-2539.

5. Nogee LM. Abnormal expression of surfactant protein $\mathrm{C}$ and lung disease. Am J Respir Cell Mol Biol 2002; 26: 641-644.

6. Nogee LM, Dunbar AE, Wert S, Askin F, Hamvas A, Whitsett A. Mutations in the surfactant protein $\mathrm{C}$ gene associated with interstitial lung disease. $N$ Engl J Med 2001; 344: 573-579.

7. Nogee LM, Dunbar AE, Wert S, Askin F, Hamvas A, Whitsett A. Mutations in the surfactant protein $\mathrm{C}$ gene associated with interstitial lung disease. Chest 2002; 121: Suppl. 3, 20S-21S.

8. Thomas AQ, Lane $\mathrm{K}$, Philips $\mathrm{J}$, et al. Heterozygosity for a surfactant protein $\mathrm{C}$ gene mutation associated with usual interstitial pneumonitis and cellular non-specific interstitial pneumonitis in one kindred. Am J Respir Crit Care Med 2002; 165: 1322-1328.

9. Tredano M, Griese M, Brasch F, et al. Mutation of SFTPC in infantile pulmonary alveolar proteinosis with or without fibrosing lung disease. Am J Med Genet 2004; 126A: 18-26.

10. Hamvas A, Nogee LM, White FV, et al. Progressive lung disease and surfactant dysfunction with a deletion in surfactant protein C gene. Am J Respir Cell Mol Biol 2004; 30: $771-776$

11. Loyd JE. Pulmonary fibrosis in families. Am J Respir Cell Mol Biol 2003; 29: Suppl. 3, S47-S50.

DOI: $10.1183 / 09031936.04 .00092304$ 